\title{
An Overview of the Available Health System for Monitoring Non-Communicable Diseases in Malawi
}

\author{
Safary $E^{1,2 *}$ and Mtaita $C^{1,3}$ \\ ${ }^{1}$ Heidelberg Institute of Global Health, Heidelberg \\ University, Heidelberg, Germany \\ ${ }^{2}$ Foundation for Innovative New Diagnostics, Geneva, \\ Switzerland \\ ${ }^{3}$ Deutsche Gesellschaft für Internationale \\ Zusammenarbeit (GIZ), Bonn, Germany \\ *Corresponding author: Elvis Safary, Heidelberg \\ Institute of Global Health, Heidelberg, 69115, Germany
}

Received: September 01, 2021; Accepted: October 01, 2021; Published: October 08, 2021

\begin{abstract}
Background: Non-Communicable Diseases (NCDs) are a major cause of morbidity and mortality in Malawi and they come second as the leading cause of death in adults after HIV and AIDS. The World Health Organization (WHO) highlighted the importance of NCD monitoring as a key action which were adopted by the Member States in addressing NCDs. The framework is composed of nine global targets and 25 indicators aimed at combating global mortality for the four main NCDs. This study aimed to examine the existing national NCD monitoring tools, activities and gaps in Malawi based on WHO Global Monitoring Framework for NCDs.
\end{abstract}

Methods: A desk review was conducted from June to August 2021, to examine the existing national NCD monitoring tool in Malawi from multiple sources. Policy and program documents relating to NCD monitoring in Malawi from 2011 to 2021 were identified and analyzed.

Results: The findings of this review are presented according to the three major themes of the Global Monitoring Framework: Health systems capacity, monitoring of risk factors, and monitoring of mortality and morbidity outcomes. Malawi has an NCD monitoring tool in place that is adequate for the WHO NCD Global Monitoring Framework. However, there are still gaps with data from monitoring health systems indicators of the framework and hence requires strengthening.

Conclusion: The country must also look beyond these set of indicators and target increasing burden and impact of COVID-19 pandemic.

Keywords: Non-communicable diseases; Malawi; COVID-19; Surveillance

\section{Introduction}

Non-Communicable Diseases (NCDs) are the leading cause of death globally [1] and are strongly influenced by four main behavioral risk factors: tobacco use, insufficient physical activity, harmful use of alcohol and unhealthy diet, which leads to elevated blood pressure, raised blood glucose and cholesterol levels and excess body weight [1]. Non-communicable diseases (NCDs) are significant causes of morbidity and mortality in Malawi and the second leading cause of deaths in adults after HIV and AIDS [2]. NCD incidence are rapidly high, according to the Malawian Ministry of Health (MOH) in 2017, NCDs in the country accounted for at least $12 \%$ of total disability adjusted life years (DALYS) and at 16\%, are the second leading cause of death after HIV/AIDS [3]. NCD disproportionally affect young people, with up to $60.5 \%$ of NCD DALYs constituting people under the age of 40 , as compared to $18.5 \%$ in high-income countries [4]. The World Health Organization (WHO) has elaborated strengthening surveillance at national level as a priority for tackling Non-Communicable Diseases (NCD) [5-7]. This is an ongoing systematic collection and analysis of data to provide appropriate information regarding a country's NCD burden, the population groups at risk, estimates the NCD mortality and morbidity, risk factors and determinants of disease over time. In 2000, the global strategy for the prevention and control of NCDs sanctioned by the World Health Assembly highlighted the surveillance to track and monitoring the major risk factors as a central priority [5]. The WHO however has also recognized that several key steps must be taken to strengthen surveillance for NCD [7]. These include prioritizing monitoring and surveillance of behavioral and metabolic risk factors in low-resource settings, components of the NCD surveillance framework, and strengthening and integrating the NCD surveillance system into existing national health information system such as the District Health Information System (DHIS). In 2012, the WHO set a comprehensive set of nine global targets and 25 indicators, [5] with the aim to ensure prevention, treatment and accountability for NCDs in WHO supported countries. Malawi is a landlocked country in Southeast Africa with an area around 120,000 square kilometers, but it is also one of the most densely populated countries in Africa with 21.2 million people [2]. Urbanization and environmental factors, low health literacy and rapid aging have contributed to steady increase in the prevalence of NCDs in Malawi [8]. Following the 2011 United nations declaration on NCDs, Malawi developed and launched the 2011-2016 Health Sector Strategic Plan including NCDs and the National Action Framework for NCDs in 2013 [9] which focused primarily on a narrower set of conditions comprised of cardiovascular disease, diabetes, chronic respiratory diseases and cancer. This was then followed by the National Action Plan for the Prevention and Management of Non-Communicable Diseases (NAP-NCD) 2017-2022 [10]. The framework expands its emphasis beyond the 
traditionally recognized NCDs and emphases an integrated approach, noting that addressing the more broad burden of NCD will be a critical step towards Universal Health Coverage (UHC) for Malawi [10]. The NAP-NCD 2017-2022 had incorporated the WHO-NCD global monitoring targets and emphasizes the precarious importance of an effective surveillance system to track, trace and construe tends in risk factor (behavioral risk factors, physiological and metabolic risk factors and social determinants), morbidity (cancer incidence and type) and mortality (NCD- specific mortality), and health systems capacity and responses (interventions and health system capacity such as infrastructure, policies and plans, access to key health-care interventions and treatment). The main objective of this study is to examine the current national NCD monitoring tools, activities and gaps in Malawi based on the Global Monitoring Framework for NCDs.

\section{Methods}

A desk review was conducted from June to August 2021, to examine the existing national NCD monitoring tool in Malawi from multiple sources. The search strategies included literature databases, search engines, and google websites. The search strategy included the following terms: Malawi, non-communicable diseases, monitoring, surveillance, guidelines, framework and standards. A search was conducted in June and July 2021 using Google scholar and PubMed. Additionally, relevant documents in the internet were also searched including a search of relevant organizations and institutions for gray literatures. Authors targeted only documents that were relevant to the research objective. Relevant policy documents, guidelines, program documents and reports were collected from suitable organizations and institutions based on the research topic. Finally, routine data including monitoring and evaluation of NCD were collected for inclusion in the review.

\section{Results}

\section{Implementation of the NCD monitoring in Malawi}

Implementation of NCD monitoring in Malawi is described in this study based on the three major themes of the WHO Global Monitoring Framework for the 25 indicators: monitoring of risk factors; health systems capacity and monitoring of outcomes.

\section{Monitoring of risk factors}

Malawi national STEPS non-communicable diseases risk factor survey, which is based on the WHO STEPs survey [11], is a population based cross-sectional survey of chronic diseases and their risk factors. It is designed to help countries build and strengthen their capacity to conduct surveillance. This survey monitored lifestyle risk factors (physical activity, tobacco and alcohol use) and anthropometric measurements (body mass index and central obesity) as monitored in the Global NCD Framework with exception of fat and salt intake. Both fat and salt intake are not routinely monitored at the population level and consequently there is no consistent data available [10].The Government of Malawi uses a standard methodology for a household survey for adults. A three-step multistage sampling method is used to produce a national representative data both at urban and rural areas of Malawi. The data is collected by face-to-face interviews using structured questionnaires as well as collection of clinical and biochemistry tests.

\section{Health systems capacity}

The Ministry of Health occasionally conducts health facility survey to enable reporting of drugs and essential technologies and medicine indicators. This is normally a paper-based stock management which can be erroneous and time consuming. There is a shortage of equipments and essential technologies as well as essential drugs in most of the health facilities in Malawi. Furthermore, the government has a subsidy of drugs in all health facilities however, patients are often asked to purchase drugs via private avenues. Malawi uses the District Health Information System (DHIS2) to collect data and transmit them electronically from the district to the national level. However, from the health facility level to the district level, data reporting is still paper-based. This is because most health facilities do not have internet connectivity and or a computer as well as electricity. Data at the health facility level are collected through paper-based system using registers, aggregated on monthly basis and then sent to the district level. This process makes data collection slow and time consuming. The Ministry of health with assistance from non-governmental organizations have developed a suite of touchscreen-based Electronic Medical Record (EMR) system used by health facilities across Malawi. The EMR system includes applications for patient registration, in-and-out patient diagnosis, maternal care,

Table 1: Monitoring of risk factor indicators.

\begin{tabular}{|c|c|c|c|}
\hline Indicators & Name of responsible agency & Period & Remarks \\
\hline Raised blood pressure & $\mathrm{MoH}$ & Monthly & Data collected through $\mathrm{MoH}$ health facilities \\
\hline Raised glucose & $\mathrm{MoH}$ & Monthly & Data collected through $\mathrm{MoH}$ health facilities \\
\hline Raised total cholesterol & $\mathrm{MoH}$ & Monthly & Data collected through $\mathrm{MoH}$ health facilities \\
\hline Tobacco use & $\mathrm{NSO}, \mathrm{MoH}$ & - & Malawi MDG Endline survey 2014 \\
\hline Salt intake & $\mathrm{NSO}, \mathrm{MoH}$ & - & Malawi MDG Endline survey 2014 \\
\hline Overweight and obesity & $\mathrm{MoH}$ & Monthly & Data collected through $\mathrm{MoH}$ health facilities \\
\hline Saturated fat & $\mathrm{MoH}$ & - & - \\
\hline Alcohol consumption & - & - & Data not collected at national level \\
\hline Alcohol related morbidity and mortality & - & - & Data not available \\
\hline Physical inactivity & - & - & Data not available \\
\hline Prevalence of heavy episodic drinking & - & - & Data not available \\
\hline
\end{tabular}

MoH: Ministry of Health; NSO: National Statistic Office; MDG: Millennium Development Goal. 
Table 2: Health systems capacity indicators

\begin{tabular}{|c|c|c|c|}
\hline Indicators & Name of responsible agency & Period & Remarks \\
\hline Essential technologies and medicines & $\mathrm{MoH}, \mathrm{PMPB}$ & - & Survey not done/data not available \\
\hline Cervical cancer screening & $\mathrm{NCR}, \mathrm{MoH}$ & Annual & $\begin{array}{c}\text { Data is gathered from the central pathology } \\
\text { lab and compiled through an electronic } \\
\text { monitoring system }\end{array}$ \\
\hline HeP B vaccinations & $\mathrm{MoH}$ & Annual & Data collected through $\mathrm{MoH}$ health facilities \\
\hline HPV vaccination & $\mathrm{MoH}$ & Annual & Data collected through $\mathrm{MoH}$ health facilities \\
\hline Access to palliative care & $\mathrm{NSO}, \mathrm{MoH}$ & - & Survey not done/data not available \\
\hline
\end{tabular}

NCR: National Cancer Registry; PMPB: Pharmacy Medicines and Poisons Board; NSO: National Statistics Office.

Table 3: Monitoring of outcome indicators.

\begin{tabular}{|l|c|c|c|}
\hline \multicolumn{1}{|c|}{ Indicators } & Name of responsible agency & Period & \multicolumn{1}{c|}{ Remarks } \\
\hline Cancer incidence (by type of cancer) & National cancer registry, MoH & - & Underreporting and delays in reporting \\
\hline Premature mortality due to NCDs & NRB & - & Lack of comprehensive death registration \\
\hline
\end{tabular}

NRB: National Registration Bureau.

and ARV [12]. This EMR suite is used in more than 60 health facilities across Malawi but there are plans to introduce it to more health facilities.

\section{Monitoring of outcomes}

The national Statistics office in Malawi is the main government body responsible for collecting and disseminating all data on premature mortality due to NCDs. However, due to insufficient data on deaths that occur outside the health facility, majority of deaths are non-medically certified. In 2007, the Malawi Government through the National Registration Bureau (NRB) initiated a paper-based village registration system for births and deaths, and this reached full country-wide coverage by 2011 . These registers were a potential source of vital statistics data at the community/grass-roots level, but the manual collection, collation and analysis of data from villages up to traditional authorities and then up to the district commissioner were almost impossible due to lack of intermediary human resources, inadequate transport and poor infrastructure [13]. A potential solution was to develop an Electronic Village Register (EVR) to transmit data through wireless networks from village headmen to the TA Chief to the District Commissioner (DC) and then to the National Registration Bureau (NRB), with this data also shared with the appropriate health facilities [13]. In response to high burden of cervical cancer, in 2004, the Sexual and Reproductive Health Directorate of the Ministry of health Malawi, implemented Human Papillomavirus (HPV) vaccination pilot project and screen-andtreat programme using Visual Inspection with Acetic Acid (VIA) and cryotherapy. The programme is integrated with family planning services and targets women aged 30-45 years and women on HIV ART. By 2011 the programme was scaled up to all central and district hospitals, most mission hospitals and some health centers and private hospitals [8]. They receive Malawi Cancer Registry monitors all cancer incidences in Malawi. The receive notifications from all data sources providers in government and private health facilities using a national standardized tool. Comprehensive cancer registration is achieved through data obtained from a combination of sources such as hospital-based cancer registry, hospital death notification forms, pathology records, notification by medical professions and health facility discharges records. All cancer cases obtained outside health facilities and organizations physician or doctor notification are crosschecked against known registry for verification [14].

\section{Discussions}

\section{Gaps and challenges in NCD monitoring including COVID-19 in Malawi}

There is currently a vigorous monitoring system for NCD surveillance in Malawi for indicators covered by the WHONCD Global Framework. However, Malawi still lacks data for the monitoring the health system indicators of the framework. For monitoring mortalities, despite the challenges reported such as human resources, inadequate transport and poor infrastructure, all deaths that occur outside a health facility can be verbally reported by the chief or local heads. Additionally, these deaths will require an autopsy in case of suspicious foul play which will sometimes change the information of the registration of death. With regards to cancer incidences and mortalities, there are possibilities of under-reporting of cancer cases in Malawi because most cancer patients tend to seek for palliative care at their homes. Other patients often seek alternative treatment options that are not medically proven. There are several studies in Africa that have shown delay in presentation and diagnosis of cancer patients due to these factors $[15,16]$. Though there are already developed technology-based practices in place to collect data for cancer screening, there still requires paper-based data entry by the healthcare provider especially at the district level. Technology is still limited and this is well-known and documented obstacle in other studies. Some well-known challenges are lack of electricity, computer and little telecommunication coverage. Regarding COVID-19, data collection and monitoring for NCD-related consequences are two major challenges caused by the movement restriction. Current reviews have shown that SARS-CoV-2 infection may affect the progression and health outcome of pre-existing chronic disorder. Evidence from other respiratory disease show that the infection can acerbate chronic NCD conditions include and are associated with functional decline in older persons [17]. The movement restrictions have caused disruption to the screening, treatment, monitoring and follow-up of NCD patients [18]. Additionally, majority of health care workers have been re-assigned to support COVID-19 response. The transfer of healthcare workers has definitely caused disruption in data collection and data entry at primary and district health facilities. There is need to look beyond the NCD-WHO Global Monitoring Framework as the current NCD monitoring tool system in many LMICs including Malawi will not be able to monitor the NCD-related consequences 
due to COVID-19 in a well-timed manner. There is also urgency to focus more on the marginalized population especially in rural areas and strengthening of surveillance of mental health issues. COVID-19 has shown a great need to address mental health problems especially among women who experience gender-based violence. Psychological, counselling and social support services through helplines have been made available during COVID-19 pandemic. However, the utility and coverage of the data is limited as most of the services are provided by non-governmental organizations. There is great need to incorporate all paper-based data to electronic format to allow ease of decision making for policy makers.

\section{Way forward}

The roll-out of Electronic Medical Record (EMR) system in all regions in Malawi could improve the availability and accuracy of data from lower health facilities up to the national levels. Healthcare facility data on morbidity, mortality and risk factors should allow patterns to be detected in real time. The data becomes a reliable repository for NCD surveillance if it can be cross-checked across all health care facilities (public and private) and provide a platform for significant local action. Data capture procedure especially in the lower-level facilities is still paper-based and missing information are usually followed retrospectively in case of missing information. This approach can be time consuming and costly. Research has shown that electronic medical records have potential to assist enhance the privacy and confidentiality, and effectiveness of healthcare services [19]. However, challenges such as lack of training, privacy issues and high cost of installation have also been cited as potential set-backs [20]. Therefore, one option moving forward could be an expanded surveillance at the primary care level can provide important information on the progress of the different aspects of NCD services and utilization on a routine basis [21]. Utilization and modification of existing technologies to closely monitor behavioral NCD risk factors can be adopted and strengthened. This will enable us to monitor the immediate impact of any intervention related to NCD being implemented. Additionally, wearable technologies such as noninvasive self-monitoring technologies hold potential to encourage optimal self-management. Furthermore, these technologies identify new NCD risk factors, intuitions into lifestyle behaviors and tracking patient engagement in self-care [22]. There is great need to incorporate and expand NCD monitoring with the use of artificial intelligence and big data solutions. However, these options remain practically not possible given the many challenges such as financial ability, lack of health care workers and limited technological use and knowledge [23]. Some of the documents that were meant to be analyzed were not readily available from government ministries responsible for policies. As such, this limited our analysis of multisectoral action in the formulation of the policies. The data we received from the various sources on the indicator of each theme might not be exhaustive because some of the data was either not available online or up-to-date.

\section{Conclusion}

NCD monitoring is a vital and important component in response to the increasing burden of NCDs affecting the quality of life of the population and health outcomes. The existing NCD monitoring tools for Malawi is able to provide most data for the indicators in the
WHO-NCD Global Monitoring Framework, however, there is great need to strengthen the NCD surveillance in planning, monitoring and evaluation of interventions and use on all alternative sources of data. The current COVID-19 pandemic and movement restrictions have shown gaps in the health care system in Malawi and hence there is great need to strengthen the NCD monitoring system in Malawi and other countries.

\section{Declaration}

Acknowledgement: The authors also acknowledge the support of the community nurses at the Lighthouse Trust, Malawi for their time and valuable contribution during this study. The authors also thank the various research working groups and teams at the Heidelberg Institute of Global Health for their critical appraisals of this study.

Author contributions: ES and CM conceived, designed the framework and prepared the initial draft. ES refined the draft paper then both authors reviewed and approved the final manuscript.

\section{References}

1. World Health Organization. The global burden of disease: 2004 update: World Health Organization. 2008.

2. Government of Malawi. Malawi health sector strategic plan 2011-2016 Moving towards equity and quality. Department of Health (ed.), Ministry of Health Lilongwe. 2011.

3. Cundale K, Wroe E, Matanje-Mwagomba BL, Muula AS, Gupta N, Berman $\mathrm{J}$, et al. Reframing noncommunicable diseases and injuries for the poorest Malawians: the Malawi National NCDI Poverty Commission. Malawi Med J. 2017; 29: 194-197.

4. Global Health Data Exchange. Institute for Health Metrics Evaluation. Global Burden of Disease Study 2017 Data Resources. 2019.

5. World Health Organization. Global action plan for the prevention and control of noncommunicable diseases 2013-2020: World Health Organization. 2013.

6. Tunstall-Pedoe H. Preventing Chronic Diseases. A Vital Investment: WHO Global Report. Geneva: World Health Organization. 2005; 200.

7. Alwan A, MacLean DR, Riley LM, d'Espaignet ET, Mathers CD, Stevens GA, et al. Monitoring and surveillance of chronic non-communicable diseases: progress and capacity in high-burden countries. Lancet. 2010; 376: 18611868.

8. Miranda JJ, Kinra S, Casas JP, Davey Smith G, Ebrahim S. Noncommunicable diseases in low-and middle-income countries: context, determinants and health policy. Trop Med Int Health. 2008; 13: 1225-1234.

9. Boudreaux C, Noble C, Coates MM, Kelley J, Abanda M, Kintu A, et al. Noncommunicable Disease (NCD) strategic plans in low-and lower-middle income Sub- Saharan Africa: framing and policy response. Glob Health Action. 2020; 13: 1805165.

10. Government of Malawi. National Action Plan (NAP) for the Prevntion and Management of Noncommunicable Diseases in Malawi, 2017-2022. Ministry of Health. 2017.

11. World Health Organization. WHO STEPS surveillance manual: the WHO STEPwise approach to chronic disease risk factor surveillance. World Health Organization. 2005

12. Msiska B, Nielsen P. Innovation in the fringes of software ecosystems: the role of socio-technical generativity. Information Technology for Development. 2018; 24: 398-421.

13. Singogo E, Kanike E, van Lettow M, Cataldo F, Zachariah R, Bissell K, et al. Village registers for vital registration in rural $\mathrm{M}$ alawi. Trop Med Int Health. 2013; 18: 1021-1024.

14. Msyamboza KP, Dzamalala C, Mdokwe C, Kamiza S, Lemerani M, Dzowela $\mathrm{T}$, et al. Burden of cancer in Malawi; common types, incidence and trends: 
national population-based cancer registry. BMC Res Notes. 2012; 5: 1-8.

15. Mohd Mujar NM, Dahlui M, Emran NA, Abdul Hadi I, Wai YY, Arulanantham $\mathrm{S}$, et al. Complementary and alternative medicine (CAM) use and delays in presentation and diagnosis of breast cancer patients in public hospitals in Malaysia. PLoS One. 2017; 12: e0176394.

16. Taib NA, Yip CH, Low WY. A grounded explanation of why women present with advanced breast cancer. World J Surg. 2014; 38: 1676-1684.

17. Palmer K, Monaco A, Kivipelto M, Onder G, Maggi S, Michel J-P, et al. The potential long-term impact of the COVID-19 outbreak on patients with noncommunicable diseases in Europe: consequences for healthy ageing. Aging Clin Exp Res. 2020; 32: 1189-1194.

18. Chang AY, Cullen MR, Harrington RA, Barry M. The impact of nove coronavirus COVID-19 on noncommunicable disease patients and health systems: a review. J Intern Med. 2021; 289: 450-462.

19. Heart T, Ben-Assuli O, Shabtai I. A review of PHR, EMR and EHR integration A more personalized healthcare and public health policy. Health Policy Technol. 2017; 6: 20- 25.
20. Enaizan O, Eneizan B, Almaaitah M, Al-Radaideh AT, Saleh AM. Effects of privacy and security on the acceptance and usage of EMR: the mediating role of trust on the basis of multiple perspectives. Inform Med Unlocked. 2020; 21: 100450.

21. Kroll M, Phalkey R, Dutta S, Shukla S, Butsch C, Bharucha E, et al. Involving private healthcare practitioners in an urban NCD sentinel surveillance system: lessons learned from Pune, India. Glob Health Action. 2016; 9: 32635.

22. Khakurel J, Pöysä S, Porras J, editors. The use of wearable devices in the workplace-a systematic literature review. International Conference on Smart Objects and Technologies for Social Good. 2016.

23. Price WN, Cohen IG. Privacy in the age of medical big data. Nat Med. 2019; 25: $37-43$. 